\title{
MODERN TRENDS IN THE TREATMENT OF INFECTIONS OF THE HAND
}

\author{
By R. P. JePson, F.R.C.S., and H. Bolton, F.R.C.S. \\ From the Surgical Professorial Unit, The Royal Infirmary, Manchester
}

The methods of treatment advocated in this paper are based on the personal experiences of the authors over the past three years. Some indication as to the frequency of hand infections at a general hospital and the material studied will be evident from the following statistics. Only the more common and important septic lesions are included.

\section{New Patients Attending the Septic Hand Clinic

$\begin{array}{lllllll}\mathbf{1 9 4 6} & . & . . & . . & \ldots & \ldots & \mathbf{1 , 6 8 0} \\ \mathbf{1 9 4 7} & . & . . & . . & . . & . . & 2,076 \\ \mathbf{1 9 4 8} & . & . & . . & . . & . . & \mathbf{1 , 8 0 6}\end{array}$

Analysis of Cases for 1948

\begin{tabular}{|c|c|c|c|}
\hline Pulp space infections & $\cdots$ & \multicolumn{2}{|c|}{24 per cent } \\
\hline Paronychia (acute and chronic) & . & 25 & $\therefore$ \\
\hline Phalangeal infections & . & 12 & ", \\
\hline Web space infections & $\cdots$ & 5 & ", \\
\hline Boils and carbuncles & $\ldots$ & 6 & ", \\
\hline $\begin{array}{l}\text { Miscellaneous (including subcu } \\
\text { fections, septic laceration } \\
\text { abrasions) }\end{array}$ & $\begin{array}{l}\text { ticular in- } \\
\text { burns, }\end{array}$ & 28 & \\
\hline Tendon sheath infections & . & 4 & cases \\
\hline Palmar space infections & . & 2 & ,' \\
\hline Erysipeloid of Rosenbach & . & 2 & 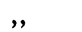 \\
\hline Average number of atten & nces & & \\
\hline $\begin{array}{c}\text { patient } \cdots \\
\text { Average number } \ddot{\text { of patients }}\end{array}$ & $\ddot{\text { attending }}$ & 7 & \\
\hline $\begin{array}{c}\text { each day } . \\
\text { Average duration of treatment }\end{array}$ & $\begin{array}{l}\cdots \\
\cdots\end{array}$ & $\begin{array}{l}44 \\
16\end{array}$ & days \\
\hline
\end{tabular}

It is important to realize that most hand infections can be avoided, aborted or brought under early control if adequate working precautions and early first-aid facilities are available. In this paper we shall deal, however, not with the prophylaxis but with the treatment of established hand infections.

\section{Pulp Space Infections (Felons)}

The frequency of these lesions and their common association with massive soft tissue necrosis and osteitis make them the most important group attending the infected hand clinic. If treatment is delayed or inadequate, a poor tactile pad and a stiff finger follow. A lateral transpulp incision is complicated in 2 per cent. of patients by a persistent painful state. The latter will be described in more detail later. Approximately $2 \frac{1}{2}-5$ per cent. of the total workmen's compensation claims are for septic hand lesions, and a considerable percentage of these follow pulp space infections. Before adequate therapy can be administered the natural history of these lesions must be understood. The organism causing the primary infection is almost always a staphylococcus aureus, coagulase positive (Bolton, Catchpole and Jepson, 1947). It is introduced into the pulp pad by a prick or cut in about half the cases and in those who can remember no local injury it is assumed to enter by way of a sweat gland. The bacteria may be transient or resident on the hand skin and a recent investigation (Moss, et al., 1948) suggests that a common source of the organism is the nasal vestibule, the contamination being by direct hand to nose contact.

Once the organism is established in the fibrofatty pulp space of the finger, a cellulitis arises during which stage the patient complains of a 'pricking' or 'tight' pain. This early inflammatory reaction is situated between the stout fibrous septa running from skin to periosteum; it may resolve spontaneously or with the aid of chemotherapy. Should resolution not occur, pus is formed.

The resulting abscess is limited by the fibrous trabeculae to one or more of the fibro-fatty compartments and involves only a portion of the pulp space. As the abscess grows it spreads in one or more of three directions:-(a) through the periosteum to involve the terminal phalanx (or occasionally round the side of the bone to present as a 'paronychia'); (b) through the skin in front forming at first a collar-stud abscess under the horny layer ; (c) through the containing fibrous walls at its sides to the rest of the pulp space (Fig. I). Usually the skin and periosteum give way first, so that a skin sinus or an osteitis arises before the whole of the pulp space is involved. If left untreated the outcome of a pulp space abscess depends therefore on its site. If superficial, a skin sinus develops and by discharging the pus and slough, may lead to a spontaneous cure. The deeper abscesses and especially the more distal 


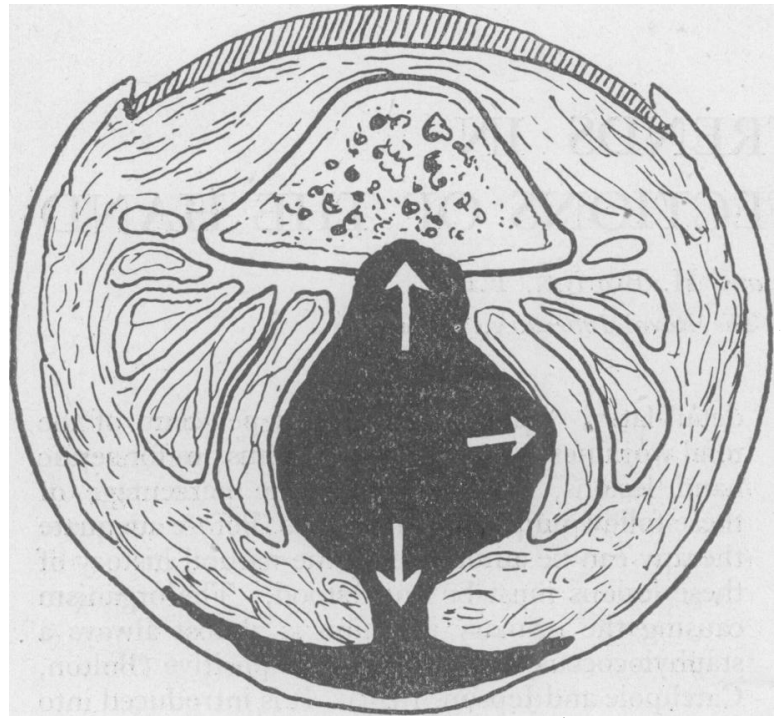

Fig. I.-The possible routes of spread of a pulp-space abscess.

ones, where there is less soft tissue cushion between the skin and periosteum, are followed by an . early periosteal necrosis and osteitis. Necrosis of the whole of the pulp space, as evidenced by fluctuation, is a late stage and it may be assumed that the phalanx is dead. In support of the above pathology is the clinical fact that the spontaneous skin sinus and the earliest $\mathrm{X}$-ray evidence of phalangeal erosion always arise directly opposite the site of the maximal abscess tension. Thus a distal pulp space abscess leads to an osteomyelitis at the tip of the phalanx, a proximal one to an initial erosion at the base (Fig. 2). We do not helieve that pressure occlusion or thrombosis of bigital vessels plays a significant part in the natural distory of a pulp space infection.

\section{Glinical Diagnosis}

There are few clinical conditions which can be confused with a pulp space infection. Gangrene of a single finger tip which is occasionally seen in arteriosclerotic patients is sometimes mistakenly incised. The lack of tenseness and local heat, together with the general evidence of arteriosclerosis should prevent this mishap.

The diagnosis once made, it is important to separate the simple felon ( $7 \circ$ per cent.) from the group which already have an established bony infection ( 30 per cent.). The latter may not be shown by $\mathrm{X}$-ray at the first attendance at the clinic ; clinical signs suggestive of bony infection must therefore be relied upon. These are :- (a) Previous inadequate incision; especially common when performed under local $\frac{2}{3}$ ethyl chloride.

(b) Spontaneous skin sinus formation. This $\frac{\varrho}{C}$ sign, first described by Klapp, is usually reliable.

(c) Massive soft tissue necrosis as demonstrated음 by 'bogginess' on palpation, 'club- $\frac{\bar{\sigma}}{\bar{x}}$ shaped ' pulp or direct operative findings. $\frac{\bar{D}}{\widehat{D}}$

(d) Post-operative pain.

\section{Treatment}

The correct treatment of a pulp-space infection $\overrightarrow{-}$ is without doubt incision and drainage as soon as $\omega$ pus has formed. Less than 5 per cent. of cases seen by us were in the 'cellulitic' stage, when 3 splintage and rest together with' chemotherapyi may occasionally abort the abscess formation. or Evidence of a local abscess is a throbbing pain and ${ }^{N}$ focal tenderness and tenseness when tested by $a+$ i match-stick or probe.

The first 400 cases seen were incised by a은 lateral 'hockey-stick' incision. This we aban-doned because (a) it is common experience that $a \subseteq$ pulp space abscess which has been widely opened through a lateral incision, will continue to drain $\vec{\ominus}$ through a tiny spontaneous skin sinus directoy overlying it, whilst the lateral incision will segl off; (b) on general surgical principles it seem logical that a local abscess should be drained by:a direct incision rather than through a valve-like slit, which opens up previously unaffected tissue and quickly closes by oedema ; (c) we have been $\stackrel{\mathbb{Q}}{2}$ impressed by the number of "persistent painful $\vec{O}$ states' which follow digital nerve injury especially 3 where sepsis is present. To avoid severing a? major digital nerve branch with a lateral incision is more fortuitous than deliberate, whilst with the응 direct incision the nerves are shouldered away by the abscess and are not involved in the incision.

In the last 18 months we have used direct incisions only.

\section{Technique of Direct Incision (Fig. 3)}

The site of the abscess must be accurately을 localized prior to incision. If a collar-stud abscess $\frac{\mathrm{N}}{\mathrm{N}}$ or a skin sinus is present, the abscess cavity is easily found. The finger must be completely N anaesthetized by a digital nerve block or a general $\mathrm{CW}_{\mathrm{J}}$ anaesthetic and the field rendered bloodless by $\mathrm{a}_{0}$ finger or arm tourniquet. An ellipse of thickened horny skin is excised over the abscess when a threatened or frank sinus is usually obvious in the cutis vera. This is incised for a few millimetres in $\square$ the long axis of the finger and the edges of the incision trimmed to expose the underlying abscess $\stackrel{\mathbb{Q}}{\Omega}$ cavity. This is circumscribed and rarely more than 


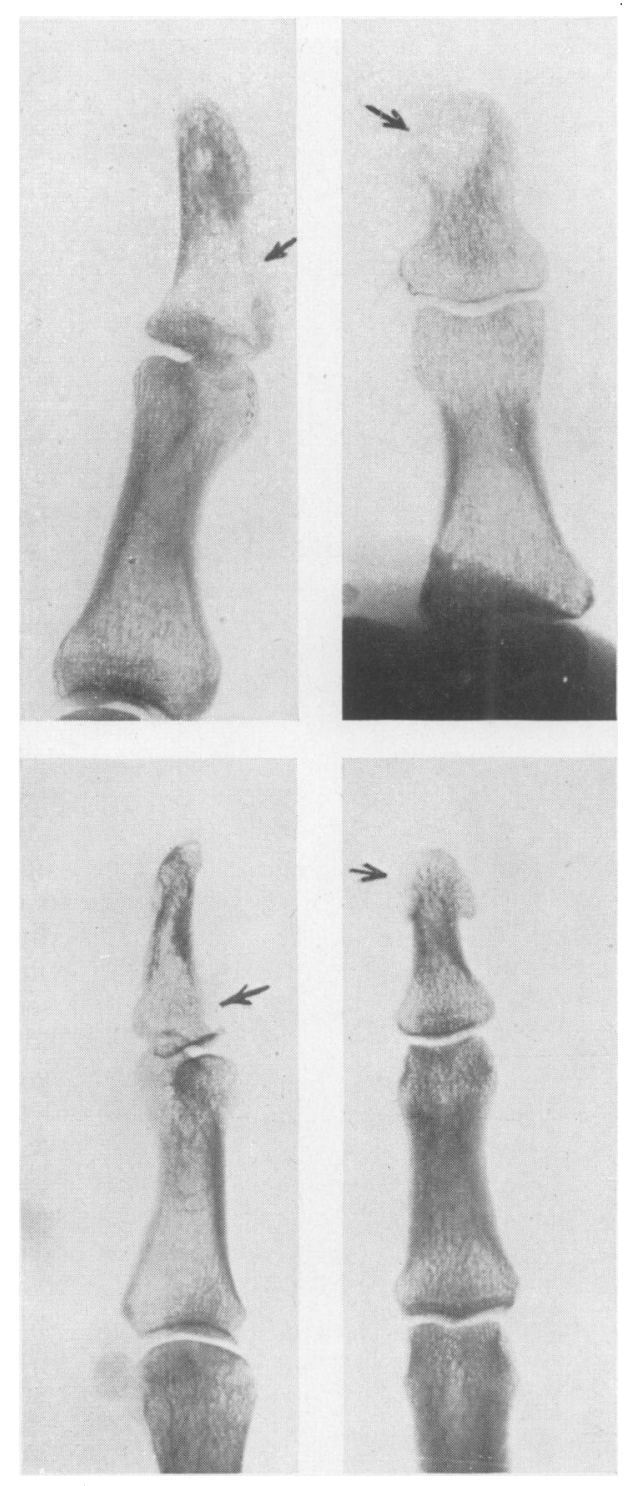

FIG. 2.- Showing that the initial bony erosion is always opposite the site of the soft tissue abscess.

a few millimetres in diameter. Apart from the evacuation of the pus and loose slough it is not disturbed. The periosteum at its base is carefully inspected for evidence of erosion. The cavity is filled with penicillin in lactose powder and the finger dressed with lightly impregnated vaseline gauze and an outer layer of absorptive dry gauze. No splint is needed, though a triangular sling for 24 hours is normally advisable. Two or three similar dressings at two to three day intervals will be sufficient, as the cavity is soon obliterated and healing occurs.

\section{The Value of Systemic Penicillin in Pulp Space Infections}

In uncomplicated pulp space infections systemic penicillin does not strikingly reduce the period of disability. It will, however, reduce the incidence of complications and should be given when lymphangitis, cellulitis, osteitis or arthritis are already present. Barber (1947) has shown that the percentage of penicillin-resistant staphylococci may be increasing and this is certainly borne out by our recent experiences. With such strains topical streptomycin $(2,000$ units/cd.) has been of value.

\section{Results}

The period of incapacity in a largeteries of pulp space infections in various clinics has been reviewed recently by Barclay (1949). In our collection the simple felon required an average of 17 days for healing with lateral and about 12 days with direct incisions. The latter technique also gives a better cosmetic result, a more painless convalescence and a lower incidence of postincisional painful states. When a suspected or established osteitis or an arthritis is present a similar surgical technique is used with the addition of systemic penicillin for Io to I4 days as an out-D patient. These cases will heal in four to six weeks, with a partial or total reformation of the terminal phalanx. We do not recommend removal of the terminal phalanx either in whole or in part, unless there is clear X-ray evidence of sequestration or avascular necrosis, both of which are uncommon.

\section{Acute; Paronychia}

' The abscess usually starts on the deep aspect of the nail fold and is at first hidden by the normal adhesion of the nail fold to the nail. It may spread to the superficial aspect of the nail fold or under the nail, but remains subcuticular' (Pilcher, I948).

Once the above pathology is understood, these infections are not a problem. In the cellulitic stage many settle with local rest and systemic penicillin therapy. Where pus has formed unilateral or bilateral nail fold incisions will be needed, and only the nail 'floating' free from its bed should be removed (Fig. 4). Because of the subcuticular site of the abscess, a skin sinus results before an osteitis can develop in the untreated case and complications are rare.

A variant of the classical paronychial infection is the chronic granuloma, called by Iselin a botryomycoma (Fig. 5). This apparently arises from a low grade infection and is treated by a wedge excision including its base. Application of 
Fig. 3 (a). Central pulp space infection with threatened sinus formation.

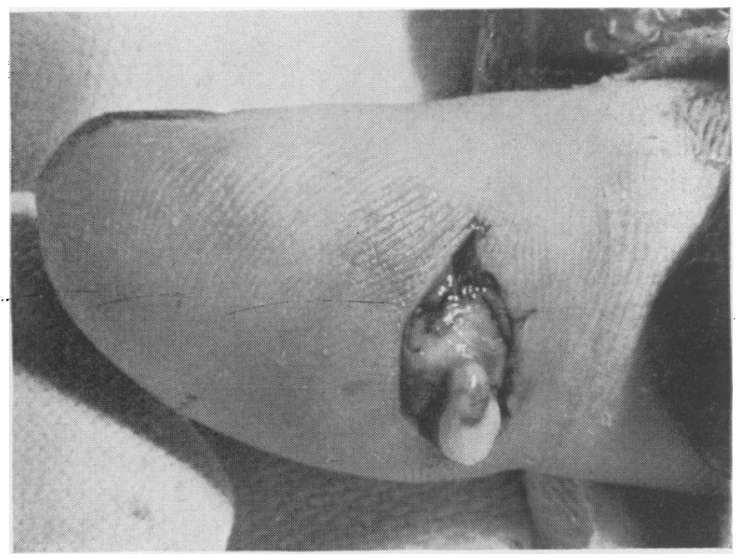

FIG. 3 (c). Showing tense localized pulp abscess.

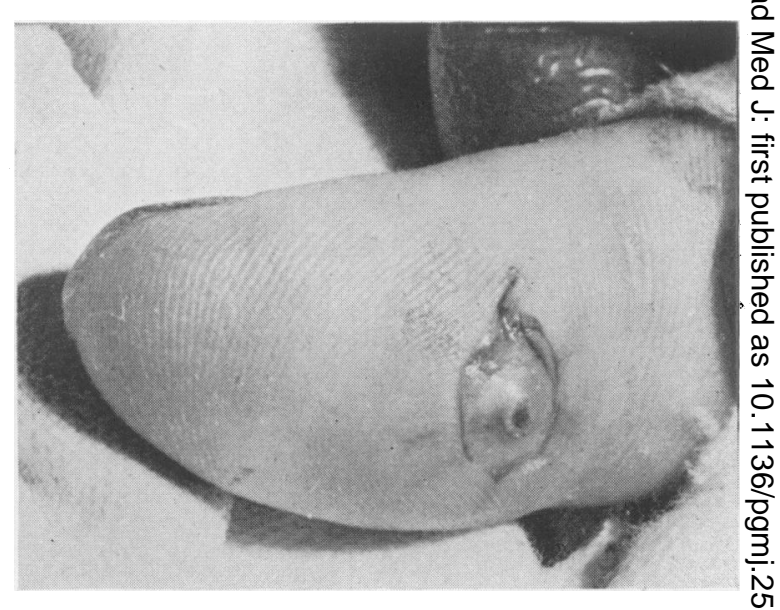

FIg. 3 (b). Transverse incision. Removal of ellipse of squamous epithelium.

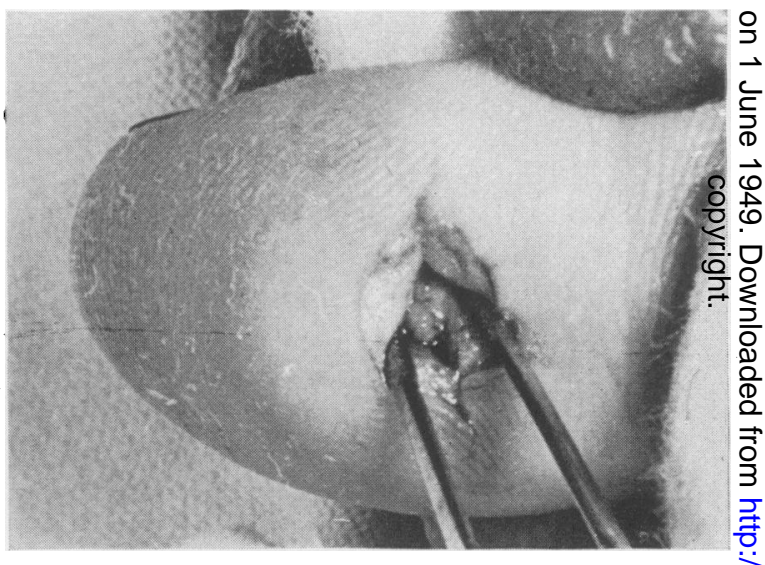

Frg. 3 (d). Abscess saucerized by removal of ellipse of true skin. One abscess extended down to intact periosteum.

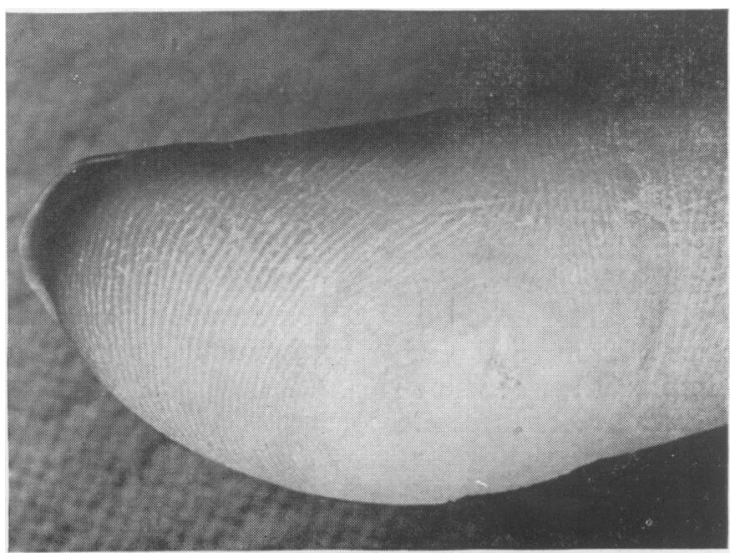

FIG. 3 (e). One week later incision healed. 


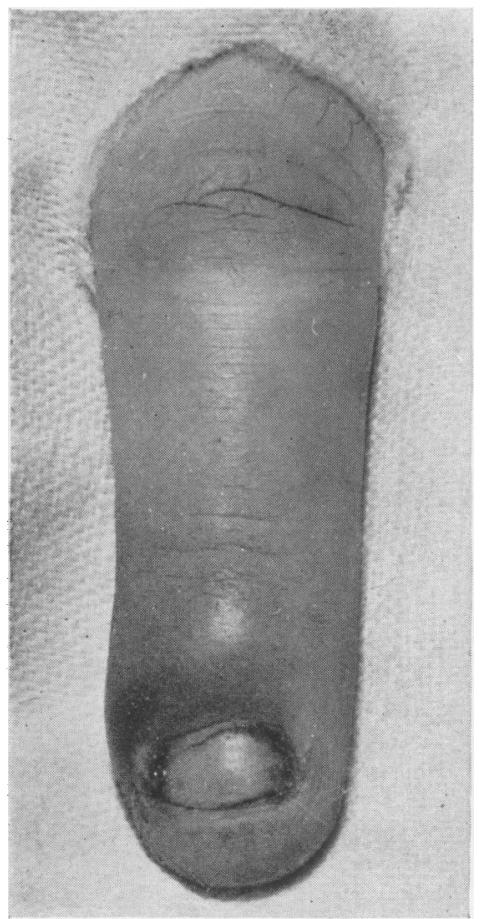

FIG. 4 (a)

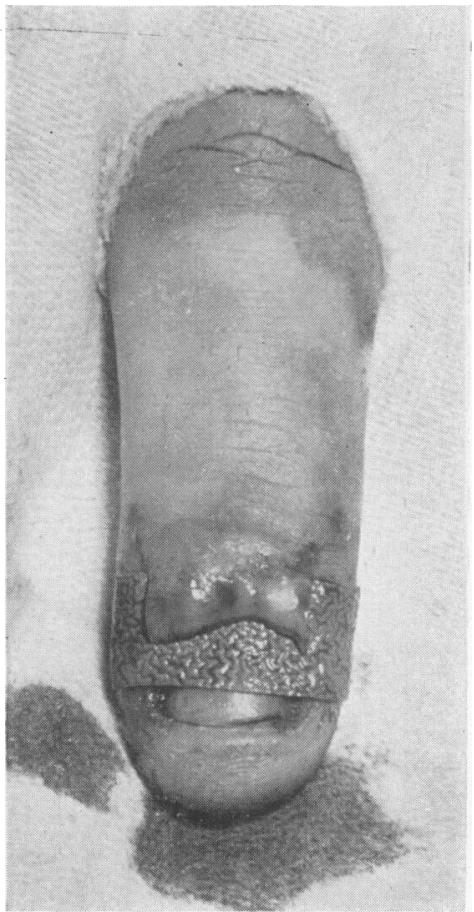

Fig. 4.-

(a) Paronychia.

(b) The incisions.

(c) The 'flouting' proximal half of nail removed.

(d) The strip of rubber glove drain in position.

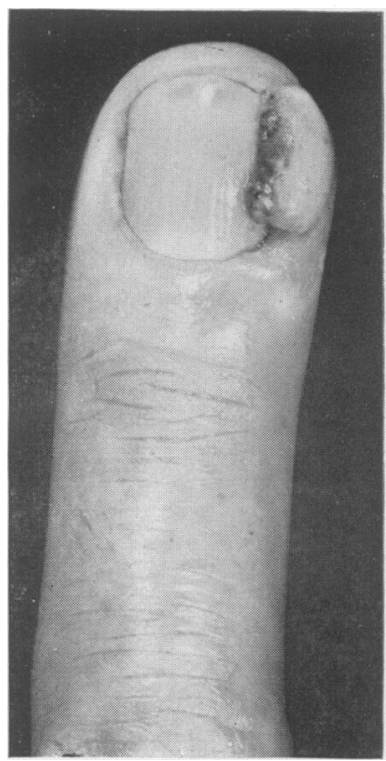

Fig. 5.

Classical Chronic Paronychia

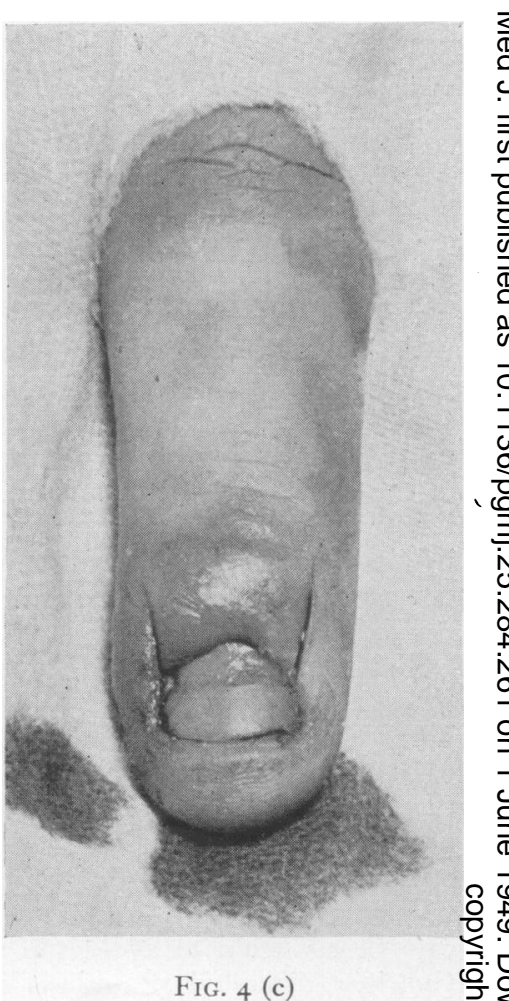

응

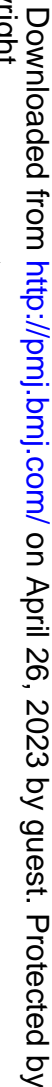


caustics or scraping is useless, the granulation tissue reformingr surprisingly rapidly.

\section{Web Space Infection}

This is a subcutaneous infection of the web space which often extends to or spreads from the loose subcutaneous space on the palmar aspect of the proximal phalanx. It is normally accompanied by considerable oedema over the dorsum of the hand and interdigital cleft and because of this may be misinterpreted as a palmar space infection.

Anatomically, the infection lies in front of the superficial palmar ligament and is limited in its more proximal spread by the increasing adherence of the skin to the palmar fascia. The web space infection never spreads more deeply into the palmar spaces, and the abscess points either in the web space or on the palmar aspect of the proximal phalanx.

When an abscess has formed it should be drained through an incision sited directly over the pus. Thus for an anterior collection a transverse skin crease incision may be used or a cleft incision for those pointing in the web space. A narrow ellipse of thick skin is excised, the abscess carefully opened through the true skin and enlarged as necessary to drain the cavity adequately, great care being taken to avoid the digital nerves and vessels. A small glove drain is left in for 24 hours. Immobilization and sometimes elevation will be necessary for two to three days. As soon as the oedema and pain have subsided, active finger exercises should be instituted.

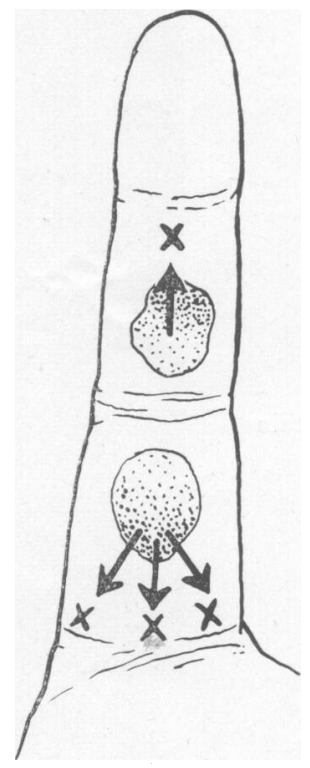

FIG. 6. The sites where phalangeal infections typically point.

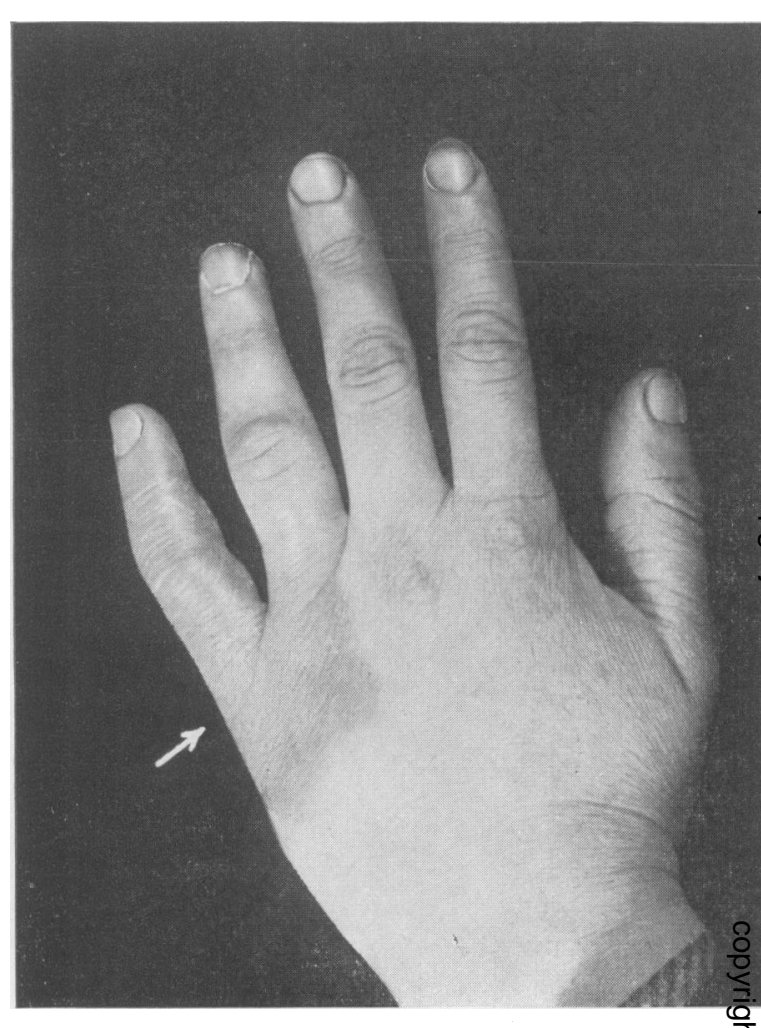

FIG. 7.-Erysipeloid of Rosenbach.
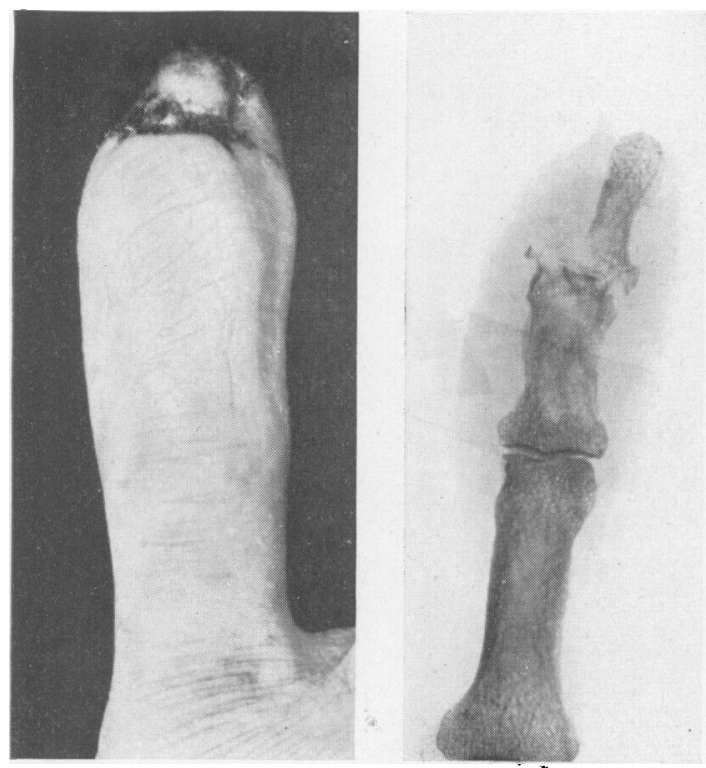

FIG. 8.-Clinical and radiological appearance of interphalangeal arthritis. 
The large thenar web with its loose subcutaneous tissue causes most confusion in diagnosis and may closely imitate the true thenar space infection. In the former the pus does not lie in front of the adductor pollicis but in the lax tissue of the web itself. When this web abscess is drained, care must be taken to avoid the aberrant radial artery, which may run in the space.

\section{Phalangeal Infections}

These are subcutaneous infections on the palmar aspects of the middle and proximal phalanges. The abscess points in one of two places depending on its original site (Fig. 6). It usually involves the subcutaneous space of one phalanx only, but a few transgress the interphalangeal flexor crease. Treatment is by drainage of the abscess at the point of maximal tension, local application of penicillin powder and immobilization for 24 to 36 hours. Complications are rare. Nerve and tendon injury may follow injudicious surgery.

\section{Tendon Sheath Infections}

Excluding the gross traumatic injuries these mainly arise by (a) a perforating wound, often a minor prick, usually opposite the flexor creases where the tendon sheath is closely bound to the skin; (b) as a sequel to a phalangeal infection ; (c) due to an injudicious surgical incision.

Diagnosis. The diffusely swollen finger is held immobile and semi-flexed. Severe pain is experienced on passive extension though the patient can usually flex the finger painlessly through a small range. The maximal tenderness is over the proximal cul-de-sac of the affected tendon sheath.

Treatment. All cases of tendon sheath infection should be admitted to hospital.

(a) Early cases. Within the first 24 to 48 hours of onset, the majority of cases settle quickly on systemic penicillin, roo, 000 units three hourly. The finger is splinted for two days, then active movements commenced.

(b) Late cases. In those cases which do not respond quickly to intensive penicillin therapy or which are seen late and it is suspected that pus has formed in the tendon sheath, a small lateral incision is made over the proximal phalanx and the synovial sheath washed out with penicillin solution using a fine plastic catheter. No attempt is made to drain the sheath, although the small skin incision is left open. Active movements within the limits of pain should be commenced after 48 hours' splintage.

Results. These are now excellent in contrast to the almost uniformly disastrous outcome in the pre-penicillin era. Except when the tendon was necrotic, all our patients have recovered adequate or complete function.

\section{Palmar Space Infections}

True palmar space infections are uncommon lesions. Hypothenar space infections should be drained through a longitudinal Henry incision along the ulnar border of the hand, thenar infections by an anterior incision in Lange's lines and the introduction of forceps. The deep middle palmar abscess necessitates the splitting of the web between the third and fourth fingers. A pair of forceps is then introduced to ensure drainage. Early active movements are imperative especially in the older age groups.

\section{Erysipeloid of Rosenbach (Fig. 7)}

This is an intra-epithelial erysipeloid of low virulence and slow progression leading to desquamation. It is important that it should be diagnosed correctly as surgery is contraindicated. A history of a prick whilst handling fish or swine is almost always obtainable and the condition is commonly seen at hand clinics serving abattoirs and meat and fish markets. One of our cases abraded his thumb whilst dissecting an elephant The infecting organism is erysipelothrix rhusiopathiae. The skin is hot and purple-red and the patient complains of a pricking or tight sensation but no pain. The sharply-defined flat edge of the infection advances slowly, rarely giving rise to lymphangitis. It has the peculiar characteristic of involving neighbouring fingers by spreading distally from their base. Suppuration never occurs and constitutional symptoms are slight or absent. The disease is self-limiting with a tendency to relapse. Serum and sulphonamides have been tried but the most useful therapy in our experience has been penicillin 200,000 units twice daily for at least seven days. This seems to clear the infection and prevent relapse.

\section{Terminal Interphalangeal Arthritis}

This should present no difficulty in diagnosis. Cases fall into two groups, (a) when an uncontrolled phalangeal osteitis following a pulp space infection spreads proximally into the joint and sometimes beyond, (b) following a paronychial infection when it betrays itself by the extensive dorsal fusiform swelling which is always present (Fig. 8). Interphalangeal joint movement is painful and restricted. The diagnosis may be clinched by X-ray and also by the following test. Clasping the middle and terminal phalanges, the surfaces of the interphalangeal joint are gently moved over each other from side to side. Abnormal mobility and palpable fine crepitus are 
diagnostic. Treatment is by drainage of the original lesion, splintage and systemic penicillin therapy for 10 to 14 days. A considerable number of joints so treated regain some useful movement.

\section{Notes on Methods and Complications}

Penicillin. This is most satisfactorily administered in an out-patient organization by a daily injection of penicillin in a delayed absorption menstruum. Clinically one must be on the outlook for the septic finger which does not respond as expected whilst on penicillin therapy. Bacteriological and penicillin sensitivity studies should be made in all severe hand infections as a routine.

Streptomycin.-No supplies for parenteral administration are yet available. Where penicillinresistant organisms are present and the degree of infection warrants its use, we have applied streptomycin locally in a concentration of 2,000 units per cc. The technique is to cover the infected area with a layer of mesh tulle gras, on which is laid several thicknesses of gauze saturated in streptomycin solution. This is covered with sterile oiled silk whose edges are sealed with mastisol. A single topical application of streptomycin retains its potency for about 48 hours, which is usually sufficient to sterilize the surface of the infected area.

Dressings. The dressings should be sterile, absorptive, painless and easily removed. A single layer of wide mesh tulle gras covered with sterile dry gauze fulfils these criteria. Soaks, moist dressings and antiseptic baths encourage secondary infection and lead to swollen oedematous fingers, exaggerating the normal inflammatory oedema.

Sterile ribbon gauze impregnated with penicillin cream is useful for large cavities. Penicillin powder is made up with lactose powder to avoid ' caking.' One-half per cent. brilliant green is a useful application in the end phase of many hand infections, where there is a superficial flat unhealed surface.
Physiotherapy. We do not use short-wave diathermy or infra-red irradiation as they mean frequent disturbance of the dressings and the risk of secondary infection. Immobilization of a finger or hand by a copious dressing of cotton wool and a sling is usually sufficient without deliberate splintage. This is maintained as long as cellulitis or lymphangitis (9 per cent. of all cases) are present. Active mobilization of the fingers within the limits of pain is of paramount importance and patients should be encouraged and instructed in hand exercises at the earliest moment. Movements are usually started 24 hours after incision and only supervised in the massage department if the patient shows hesitancy or slow progress at follow-up visits.

Persistent painful states following digital nerve injury. Similar cases have been described by Homans as ' minor causalgias.' They present with either spontaneous pain, cutaneous burning or a deep ache, or with a markedly lowered threshold to mechanical stimuli. The affected finger is cold, blue and tapering. The milder varieties which have some tactile hyperpathia and 'cold ache,' clear spontaneously aided by physiotherapy and wax baths. The severer states are extremely troublesome and require careful analysis before therapy can be instituted.

\section{ACKNOWLEDGMENTS}

Our thanks are due to Professor A. M. Boyd under whose supervision the study has been made, and to the radiological, pathological and physiotherapeutic departments of the Manchester Royal Infirmary for their technical assistance.

\section{BIBLIOGRAPHY}

BARBER, M. (1947), Brit. Med. F., 2, 863.

BARCLAY, G. A. (1949), Brit. Med. F., x, 175.

BOLTON, H., CATCHPOLE, B. N., and JEPSON, R. P. (1947) Lancet, 2, 608 :

HENRY, A. K. (1945), 'Extensile Exposure applied to Limb Surgery,' E. \& S. Livingstone Ltd., Edinburgh.

HOMANS, J. (1940), New England F. of Medicine, $220,870$.

KLAPP, quoted ISELIN, M. (1940), 'Surgery of the Hand, London.

MOSS, B., et al. (1948), Lancet, 1, 320.

PILCHER, R. S. (1948), Lancet, $1,777$. 